\title{
Resistance Training and Youth
}

\section{William J. Kraemer, Andrew C. Fry, Peter N. Frykman, Brian Conroy, and Jay Hoffman}

\begin{abstract}
The use of resistance training for children has increased in popularity and interest. It appears that children are capable of voluntary strength gains. Exercise prescription in younger populations is critical and requires certain program variables to be altered from adult perspectives. Individualization is vital, as the rate of physiological maturation has an impact on the adaptations that occur. The major difference in programs for children is the use of lighter loads (i.e., > 6 RM loads). It appears that longer duration programs (i.e., 10-20 wks) are better for observing training adaptations. This may be due to the fact that it takes more exercise to stimulate adaptational mechanisms related to strength performance beyond that of normal growth rates. The risk of injury appears low during participation in a resistance training program, and this risk is minimized with proper supervision and instruction. Furthermore, with the incidence of injury in youth sports, participation in a resistance training program may provide a protective advantage in one's preparation for sports participation.
\end{abstract}

Over the past 10 years resistance exercise training has had increased exposure and has become popular, which in turn has led to its acceptance among adults as a method to improve certain aspects of physical fitness. Greater acceptability, combined with a recent concern for youth fitness, has led to a closer examination of the efficacy of resistance training for youth.

Initially a number of perceived benefits were hypothesized to make participation in resistance training worthwhile for younger populations $(21,34)$. These included (a) increased muscular strength and local muscular endurance, (b) improved performance in sports and recreational activities, and (c) injury prevention during participation in sports and recreational activities.

This paper was developed from a presentation at the 1989 annual meeting of the American College of Sports Medicine in Baltimore, in a symposium titled "Training Adaptations and Cautions in Pre- and Post-Pubescent Children" organized and chaired by Bo Fernhall.

W.J. Kraemer and A.C. Fry are with the Center for Sports Medicine, The Pennsylvania State University, University Park, PA 16802. B. Conroy and J. Hoffman are with the Human Performance Laboratory, Department of Sport, Leisure and Exercise Sciences, The University of Connecticut, Storrs, CT 06269. P.N. Frykman is presently with the Exercise Physiology Division, USARIEM, Natick, MA 01760. 
Concomitant with the increased popular interest and scientific curiosity concerning youth and resistance exercise, professional organizations such as the National Strength and Conditioning Association, the American Orthopedic Society for Sports Medicine, and the American Academy of Pediatrics all began studying and evaluating this issue. Consequently, position papers and opinions concerning this topic have been published $(1,14,36)$.

Subsequent to this effort, a number of investigators have undertaken an intense examination of the current scientific literature $(2,17,35,45,49,55)$. From this work the following is apparent: (a) Professional organizations support the supervised use and proper exercise prescription of resistance training for prepubescent and pubescent children. (b) Only a limited number of studies are available to answer the many questions concerning exercise prescription for prepubescent and postpubescent children. (c) There is a need for a paradigm from which to develop further studies and help in the prudent recommendations for future scientific investigations.

Thus the purpose of this paper will be to examine the literature, develop an exercise prescription paradigm, and examine some of the concerns when prescribing exercise for prepubescent and postpubescent children. It is our hope that this type of review will stimulate a more sophisticated understanding and perspective concerning resistance training in youth and also help stimulate research that can bridge the gaps in our understanding.

\section{Exercise Prescription Overview}

The prescription of resistance exercise can be quantified by the use of a variable structure that calls for a decision making process in the development of a single exercise session and that changes over time $(17,27)$. The acute program variables that describe a single exercise session are choice of exercise, order of exercise, length of rest period, number of sets, and the load used. Each variable needs to be addressed in the exercise prescription process for children. Changes in the program over time are related to the chronic manipulations of these acute program variables. This usually involves variations in these variables and changes in the volume of exercise (Sets $\times$ Repetitions $\times$ Load). Various periodization methods have been proposed for changing the exercise stimulus and thereby enhancing physiological adaptations and keeping the interest in training high (17, $28,51)$. Unfortunately, scant data are available concerning the efficacy of periodization of training in children (51).

It is important to remember that adult programs should not be imposed on children. Development of a program is a cooperative effort, and the child should be an active partner in the exercise prescription process. Many times the child is left out of this process. It is important for the parent, teacher, or coach to understand, appreciate, and be sensitive to how the child feels about the training program. This important information may often result in the modification of a program, especially in the early stages. Thus, individualization of exercise programs may be even more vital to the exercise prescription process in children, due to differences in physiological and psychological maturation. Generalized programs should always be viewed as a starter point. The exercise prescription process is a dynamic activity, not a static one that is crystallized on paper.

One should know that the child understands the following: 
1. Understands the purposes of the training program;

2. Wants to try it in order to gain specific benefits;

3. Understands that he or she has control of what he/she is doing and that the supervisor will listen to his/her fears, questions, and concern;

4. Understands that training and fitness is a lifetime pursuit and that muscle strength and endurance are part of a total conditioning program;

5. Understands that the supervisor will work with him or her to develop and change the program to address his/her individual needs.

\section{Choice of Exercise}

The most important factors in the choice of exercise are that the child fits the equipment, the skill and technique required to perform the exercises are properly taught, and exercises are chosen to include major muscle groups and both agonist and antagonist muscles of the joint.

Equipment is often built for the normative adult male and requires the starting position to be altered. For example, in the bench press the bar may be 4 or 5 inches above the chest and require a block to raise the bench so that the bar is at chest level. Sometimes equipment manipulations cannot be made and an alternate mode of exercise must be chosen. A wide variety of equipment can be used if taught properly and fitted to the child's body size. Here again is another important reason for individualization of training programs: All children do not fit all equipment. Thus many types of equipment can be used, from free weights to various machines, if the "fit" criteria are utilized.

To date, no type of exercise equipment has been deemed superior (17). Increases in strength, which will be discussed later, have been observed from the use of several types of equipment, with no injuries reported in the studies. Detailed discussions of the various types of resistance exercise training modalities have been published $(17,45)$. Still, one factor that has become an important point of scientific study is the role of the eccentric contraction in development of muscular hypertrophy $(17,38)$. How important this factor is where children are concerned requires further study since certain equipment choices do not have an eccentric component.

The likelihood of injury is low with resistance training $(1,17,34)$. Certain lifts may have a higher probability of injury (17), but if proper teaching and technique are used the chances are diminished. Overhead lifts (e.g., military press) and structural lifts (e.g., squat) have been points of concern due to the possibility of injury (17). These concerns can be minimized by (a) not prematurely overloading the movement, (b) proper teaching progressions in the lifts, and (c) proper safety spotting during lifts. It appears that resistance training can be used safely if properly prescribed and supervised during the training $(35,39,40,45,46$, 47, 56).

\section{Order of Exercise}

Virtually no data exist regarding the optimal order of exercise. But common practice is to exercise large muscle groups first and then smaller ones. Furthermore, exercise progression during a workout is typically sequenced going from arm to leg (or non-arm) exercises. Changes in these sequences may increase the stress levels of the exercise session and should be done only in advanced program progressions $(17,28)$. 


\section{Rest Period Length}

The length of the rest period between sets and exercises is an important determinant of the primary energy sources during a resistance training session. High blood lactate concentrations ( 8 to $22 \mathrm{mmol} / \mathrm{l}$ ) have been observed consequent to resistance exercise protocols that utilize short rest periods $(17,30)$. It is apparent that when rest periods are extremely short and intensities are relatively heavy (i.e., 10 RM loads), only trained individuals do not exhibit symptoms of extreme fatigue and nausea (30). Still, Weltman et al. (56) have shown that short rest periods (i.e., $30 \mathrm{sec}$ ) can be used with children performing multiple-set hydraulic resistance exercise protocols in which up to 30 repetitions can be performed per set. It might be hypothesized that many of the physiological adaptations observed consequent to this style of training are due to the short rest periods used.

The importance of quantifying rest period length is slowly becoming an important consideration in the design of a strength training program. Longer rest periods (2-5 $\mathrm{min})$ are typically used when heavier weights are being lifted or during the learning phases of an exercise (17). Being aware of the stress associated with certain program variables will reduce the chances of prescribing programs that produce inappropriate stresses on children. In contrast to various hydraulic resistance exercise protocols, when heavier loads are being lifted ( 8 to $10 \mathrm{RM}$ ) using free weights or stack load machines, a gradual progression to shorter rest periods is required. Furthermore, there should be a strong rationale (e.g., preparing young wrestlers who will be exposed to high lactate environments in their sport) for incorporating such stress into the program design.

The physiological impact of short-rest resistance exercise protocols is only beginning to surface. Such metabolically stressful protocols may well help augment muscle hypertrophy due to their stimulatory effects on various endogenous anabolic hormonal and growth factor systems $(26,30)$, yet how these possible mechanisms operate in children, if at all, remains to be demonstrated.

\section{Number of Sets}

The number of sets is intimately involved with the volume of the exercise stress in resistance training. Apart from the load that is being used for the exercise, the volume of work appears to be an important variable in training adaptations related to strength and hypertrophy of muscle. Thus the number of sets allows for the progressive overload of the exercise volume as the training continues. Single-set protocols are typically performed in the early phases of training (i.e., first 2-3 weeks) when the skill development and conditioning base is very low. Then gradually the volume of exercise is increased by performing multiple sets of the exercises (e.g., three or four sets). The differential effects of various volumes of resistance training over different durations of training time have not been studied in children. The lack of effects in certain exercise training studies could be due to an inadequate exercise volume.

\section{The Load Utilized}

The load utilized in performing resistance exercise has been a primary point of concern. Some have feared that maximal or near maximal lifting performed consistently during training could increase the probability of structural injury in children $(17,40,45)$. Thus the various position stands have recommended loads 
not heavier than a 6-repetition maximum (RM) $(1,36)$. Each set would be typically targeted for at least 8 to 10 repetitions. This gives a safety zone of at least 2 repetitions.

Employing a modified periodization of load with children allows for a variation in the loading over a training period. For example, a heavy load might be listed as 8-10 RM, a moderate load as $12-15$ RM, and a light load as 18-20 RM. Using some type of variation in loading, exercises used, rest period lengths, and volume of exercise and/or exercise order may help keep the adaptations optimal and the program interesting, which in turn may increase adherence and teach children that things change as one progresses. Little is known concerning the role of program variation in the physiological and psychological changes that occur with training $(17,51)$, yet it has been shown to have great merit in resistance training for strength and power activities (51). Modifications on the concept due to differential program requirements of children are necessary. An extensive description of periodization theory and modifications for children is beyond the scope of this paper but can be found in several other sources based on the program requirements for children $(17,19,51)$.

\section{Strength Gains}

Can children improve strength? This question has been a primary issue and the subject of several reviews $(2,14,17,35,45,49,55)$. It has been generally accepted that strength training can increase strength beyond what would occur consequent to the normal growth of a child $(3,4,9,13,18,33,37,39,44,46$, $47,54,56)$. Except for some isometric training protocols $(17,22)$, almost all resistance training programs have realized strength gains in some of the musculature that was trained in the study. Still, questions remain where the lack of an effect was observed consequent to resistance training. Most of the answers may be related to factors involved with exercise prescription and testing protocols used to evaluate the training related changes.

For dynamic resistance exercise programs, a primary factor in observing more definitive overall changes in strength profiles seems to be the duration of the training period. Studies have ranged from 5 to 20 weeks and it is apparent that the longer durations of training result in significantly greater strength changes. Training adaptations may need a prolonged period of time in order to differentiate their performance contributions from those of normal growth and developmental changes. This is most likely due to the fact that the many physiological changes related to growth and development of cellular and organ systems are taking place at a rapid rate during youth. Furthermore, highly differential rates of maturation occur even within children who are matched for age $(32,43,53)$. Thus additional studies with subjects matched for maturation stage, in addition to gender, are needed to reduce the variances resulting from the differential rates of maturation.

Another confounding variable that contributes to a very conservative evaluation of strength changes in children is the difference between training and testing modalities for maximal muscle strength. Furthermore, except for a few reported studies, muscle strength is rarely evaluated using more classic 1-RM testing methods on the equipment used in training. Obviously some types of equipment (e.g., hydraulic machines) are typically unable to evaluate maximal 1-RM lifts, and thus isokinetic testing is employed for evaluation of strength changes. This part of the evaluation process has been problematic due to fear of injury 
in maximal lifts used to evaluate training changes, and to a lack of time and philosophical support in most studies to teach, practice, and determine the efficacy and reliability of maximal strength tests.

The whole issue of strength testing in prepubescent and postpubescent children needs to be examined. Many studies have not directly evaluated maximal 1-RM force production capabilities. This issue requires further study and evaluation, as most of the forces and contraction characteristics (e.g., eccentric contractions) observed in children's sports are related to dynamic concentric and eccentric force production movements, which are much greater in both exposure time and force than are properly supervised and critically chosen maximal strength tests done only to evaluate changes in muscular strength consequent to training (19).

\section{Physiological Mechanisms for Strength Development}

In an eloquent discussion of the underlying mechanisms of strength expression in children, Sale (45) describes the interplay of both tissue hypertrophy and neural factor adaptations. As training time progresses, there is an interaction between these two factors concerning the relative contributions. In children it appears that strength changes are more related to changes in the neural factors than to muscular hypertrophy.

A recent study utilizing image analysis evaluation of muscle size was unable to demonstrate increased muscle size despite significant gains in muscle strength $(4,45)$. As Sale points out, it appears that children, especially prepubescents, have more difficulty in increasing muscle size.

Thus a number of factors are responsible for the increased strength levels observed through the pubertal years (32). Strength changes typically range anywhere from 5 to $40 \%$, depending on whether the learning effect has been controlled for in the study.

It is during puberty that genital development is readily observed (32), which corresponds to increased levels of testicular testosterone secretion in males (8). This hormonal influence is most often associated with considerable increases in muscle tissue as reflected by fat-free mass $(32,48)$. The resulting progression of fat-free mass closely reflects the testosterone increases observed (41) (see Figure 1). Other hormones and growth factors intimately involved with growth and development (e.g., growth hormone, insulin-like growth factors) might be hypothesized to be compensatory factors that augment the muscular development in females (26).

Although the hormonal influence on muscle tissue is important in developing the potential for muscular strength, neuromuscular interactions are also essential for the functional development of muscle tissue $(20,42,52)$. The development of neural myelination progresses from the brain to the periphery (53), with a majority of myelination occurring by 2 years of age $(32,53)$. However, this process is not complete until sexual maturity (24) or even until adulthood (58). Myelination characteristics of a nerve are closely related to its electrical activity, affecting conduction velocity and frequency of impulse (52). Furthermore, the progression of physical capabilities in a developing child corresponds with neural myelinization patterns, as he or she is first able to control head movements, and later the arms, and finally the legs (43).

As shown in Figure 1, the theoretical curve for fiber differentiation lags 


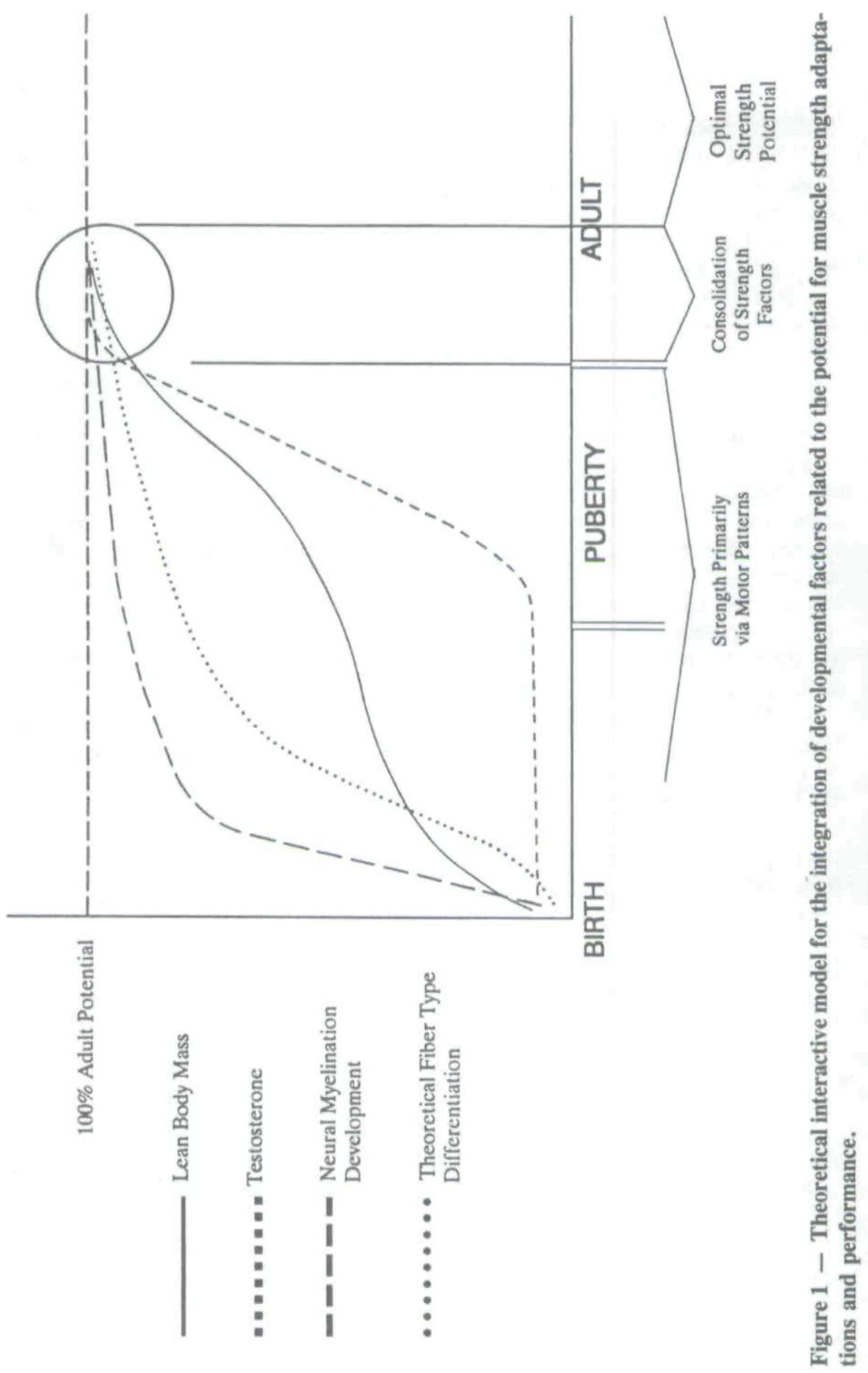


slightly behind the neural developmental curve. Again, functional development of the muscle tissue is dependent on its innervation and is augmented by the neuroendocrine system and nutritional status $(20,26,42,52)$. It is interesting to note that at birth all muscle fibers observed exhibit similar contraction times (10) and force-velocity curves $(10,12)$, indicating a lack of fiber type differentiation at this stage. Other studies have shown that the type of innervation can greatly influence the contractile characteristics of a muscle fiber $(5,6,7,11)$, thus illustrating the importance of the neural input on the developing fiber. It has been suggested that electrical activity and neurotrophic factors are both required for muscle tissue development (15). The axoplasmic flow of neurotrophic substances may be stimulated by electrical activity of the nerve $(25,31)$. However, neurotrophic factors can be received by muscle tissue in the absence of electrical activity (23).

Whether neurotrophic factors are responsible for fiber type differentiation or not, innervation characteristics are $(5,6,7,11)$. If mature innervation of muscle requires complete myelination, then the mature contractile characteristics are dependent on complete myelination. Only when each factor for strength (e.g., lean body mass, hormonal responses, neural and fiber development) has approached adult levels is the full potential array of strategies available to the individual. Thus the adaptational options for children are on a continual course of change until adulthood is reached (18). Training adaptations interact with the available physiological plasticity at each age of development, and the magnitude of adaptational change is dependent on the effectiveness of the exercise stimulus.

Little is known about how these different factors interact at various ages in response to resistance exercise training. The neural factors in prepubescent children would predominate. As the child reaches postpubescence the hormonal influences upon growth and development of muscle hypertrophy would increase. Given the scant data, one can only speculate as to the percentage of contributions from each mechanism proposed in this paper. Further research is needed to clarify the exact contributions of such physiological mechanisms interacting with resistance training adaptations in children.

\section{Other Training Benefits}

Other physiological benefits to the child have been hypothesized consequent to resistance training and they are discussed in detail elsewhere $(2,9,14,55,56)$. Briefly, improvements in body composition and blood lipid profiles have been observed $(56,57)$. It appears that physical exercise may enhance the growth rate at a given stage of development but not affect the genotypic maximum (16). Improvements in local muscular endurance and flexibility also appear to be benefits derived from participation in a resistance training program $(2,17)$. It is possible that a chronic training program would enhance bone and connective tissue development in children (17).

Improving athletic performance has been less studied due to the difficult nature of evaluating such a multivariate gestalt. Empirically, most professionals agree that training plays an important role in the preparation for sport participation $(17,18,45)$. Improvements in vertical jump have been observed as a result of resistance training $(18,45,56)$. Improving specific components of the sport skill profile is thought to enhance overall performance. Resistance training's 
greatest value might be in its preparation of the young child's body for sport participation in which the forces involved are typically high and the risk of injury is present $(34,50)$.

\section{Injury Prevention}

For a long time the fear of injury during resistance training was a contributing factor in not allowing children to participate in such training. Injury to the growth plates and the back appear to be the most dreaded. A detailed description of possible injuries is found elsewhere $(17,50)$. To date, the risk of injury consequent to appropriate resistance training programs is very low $(1,36,40,45,55,56)$. Furthermore, these isolated cases of injury may have been eliminated with proper exercise prescription, exercise techniques, and/or a reduction or elimination of the exposure to maximal or near maximal loadings of the exercise. Thus, due to the higher risk of sport injuries and the protective nature of resistance training, participation in a resistance training program would be advantageous. Limited data suggest that resistance training may have a protective value against injury $(21,35,47)$. Therefore, in addition to health and fitness benefits, participation in a resistance training program may be quite prudent especially when children are involved in sports.

\section{Integration Into a Total Fitness Program}

As has been recommended by professionals and others, resistance training should be but one part of a total strength and conditioning program $(1,17,36)$. Other components in addition to resistance exercise are generally recommended to be related to (a) aerobic, cardiovascular conditioning; (b) warm-up, cool-down, and flexibility; (c) sport, recreational activity, and skill development; and (d) nutritional status and body composition.

It is important that specific goals be discussed and that the child's schedule of activities allow for physical training in order to prepare for sports participation. This often requires a reduction in the number of sports during a season. For example, a child may not want to handle both a soccer and a baseball season simultaneously or try to play a sport during every season, as this makes a weekly schedule very difficult to manage. There must be time for sport preparatory training. Many young athletes attempt to "play" themselves into shape. This may work in some aspects of sport conditioning but it is more difficult to gain the specific benefits of resistance training. Thus some form of resistance training should be incorporated into even an in-season program. For example, although not ideal, a partner-resisted and body weight resistance program could be used (17). The bottom line is that a commonsense sports-specific resistance training and conditioning program must be implemented as part of sport participation.

\section{Program Considerations}

Before starting any sport or fitness program, the child should be cleared medically for such participation. It is important for the coach, instructor, or parent to be somewhat knowledgeable concerning the basics of resistance training. Cer- 
tification is available to professionals who desire to work in this area, and these individuals should become certified as strength and conditioning specialists (CSCS) by the National Strength and Conditioning Association. For self-education, several books and articles detail the exercise prescription of resistance training $(17,19$, 27, 28, 29, 51).

With any training program, individualization, proper progression, specificity, and variation are basic concepts that should be observed. The individual's ability to tolerate and recover from the exercise session is also important. Often the exercise prescription is too severe for the child's fitness level and recovery is not adequate. This happens when the individuals working with younger children do not have the training, intuition, or background to prescribe resistance exercise programs.

As previously mentioned, individualization appears to be vital, especially for younger children. Thus any general program prescription guidelines should be just that-guidelines-a possible starting point from which to work. Evaluate the mental and physical responses to an exercise session and be ready to reduce the exercise demands. It is always better to "'undershoot'" the child's physical ability to tolerate an exercise stress. With children it might be wiser to intentionally undershoot the physical demands, as this provides a good time for teaching lifts and allowing the child to become familiar and involved with the exercise protocols without undue fatigue. Furthermore, because children are so resilient, chronic fatigue is difficult to determine. Therefore allow 2 to 4 weeks for the child to adapt to a basic resistance training program consisting of just a limited number of sets (one or two sets), basic exercises, moderate loads (12-15 RM) and adequate recovery ( $48 \mathrm{hrs})$. This is important whether it is the first time an individual has weight trained or a new program is being started after weeks or months of no training.

Following are checkpoints for a resistance training program:

1. Parent and child both understand the reasons and benefits, and consent to participation in a resistance training program.

2. Children understand that the program is developed with them, it is only a starting point, and their responses about it are important.

3. There is competent exercise instruction, exercise technique, and safety spotting practices.

4. There is safe equipment with proper positioning.

5. Children know it is okay to tell their parents or instructor how they feel about an exercise session and that something positive will be done in response to their concerns.

Children can benefit from participation in a properly prescribed resistance exercise program. This includes those with mental or physical handicaps whose physical fitness may be low due to inactivity. The program should begin with a conservative approach and then change in response to the needs of the growing child. Few differences have been observed in the prescription of resistance exercise in females except for a need to address upper body strength requirements, which have been found to be lower than in males (17). Again, with individualized exercise prescription these needs can be addressed in the evaluation process. 


\section{Example Programs}

One should start with a basic program for 2 to 4 weeks that consists of one or two sets of each exercise. An example starter program would be as follows:

- Leg press

- Bench press

- Leg curls

- Arm curls

- Leg extensions

- Military press

- Bent leg sit-ups

- Reverse sit-ups

- Rest period length:
Three sets $10-15$ repetitions maximum same

same

same

same

same

same

same

2-3 minutes

An example intermediate to advanced program could be as follows:
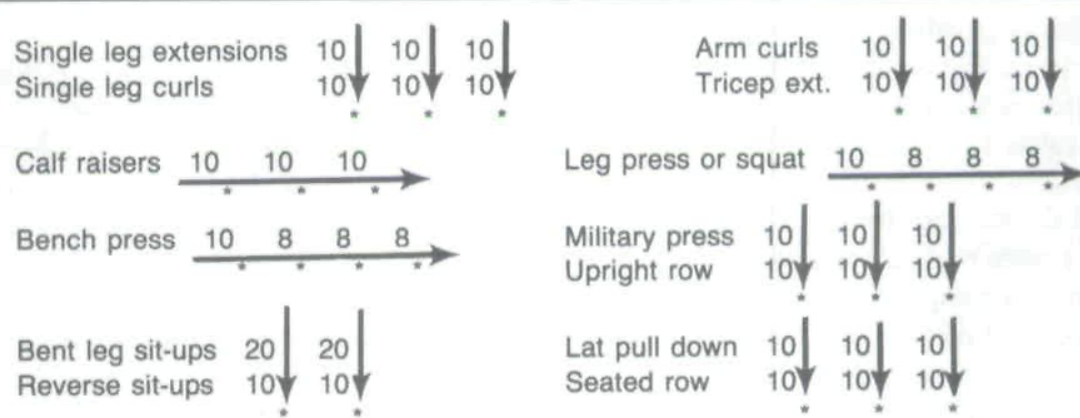

Leg press or squat $\stackrel{10 \quad 8 \quad 8 \quad 8}{\longrightarrow}$ \begin{tabular}{ll|l|l} 
Military press & 10 & 10 & 10 \\
Upright row & $10 !$ & $10 !$ & $10 !$
\end{tabular} \begin{tabular}{ll|l|l} 
Lat pull down & $10 \mid$ & 10 & 10 \\
Seated row & $10 \downarrow$ & $10 \nmid$ & $10 !$
\end{tabular}

(Additional exercises added here depending on the child's experience, exercise toleration, and training background, for example, other multiple joint exercises such as the deadlift or various pulling exercises. Three to four sets of 8 to 10.)

Figure 2 - Example of an intermediate to advanced resistance training program for children. This program requires previous training background and progression into the higher volume exercise protocols. Numbers represent sets and repetition maximum loads, while the * indicates the rest period (about $2 \mathrm{~min}$ ) and the arrows show the exercise order. One can start with any exercise or exercise group. Typical progression is from arm to leg or non-arm exercise.

\section{Characteristics of Various Program Phases}

Base Programs. These are low intensity (12-15 RM); emphasis is on teaching; recovery ability is monitored; child starts with simple lifts; volume of exercise is low (usually achieved through the number of sets and exercises in the program); duration ranges from 2 to 6 weeks; progression to intermediate 
programs is based on the needs of the child; rest periods are 2-3 minutes or more to allow for teaching and low level metabolic stress; frequency is two to three times per week.

Intermediate Programs. Higher intensities are introduced (10-12 RM); emphasis is on introducing and teaching multijoint lifts where loading is still kept light ( $>15-\mathrm{RM}$ loads); the volume of exercise (by number of sets and exercises) is gradually progressed according to the child's needs; program is balanced with other conditioning and sport schedule demands; rest period manipulations are evaluated relative to sport needs; frequency is three times per week; there is careful evaluation of recovery and toleration, thus training periods are kept relatively constant with careful manipulation of program variables; program can range from 8 to 24 weeks.

Advanced Programs. Heavier intensities (8-10 RM) are introduced; load can be periodized for major muscle group exercises; the volume of exercise is higher and is a manipulated variable within the periodization prescription, thus variation in the program over time is vital to the individualized exercise prescription process; there is greater emphasis on safety, technique, and skill in multijoint lifts; progression is based upon the child's needs; more experienced teaching and coaching is required; short rest protocols using the heavier loads can now be developed for phases of the training that have sports-specific needs; the program can be balanced with other conditioning and sport time schedules. More research is needed in this area before more definitive recommendations for exercise prescriptions for children can be made. Still, it is apparent that resistance training can play a vital role in the development of certain aspects of physical fitness for sports and lifetime health.

\section{References}

1. American Orthopaedic Society for Sports Medicine. Proceedings of the Conference on Strength Training and the Prepubescent, B. Cahill (Ed.). Chicago: Author, 1988.

2. Bar-Or, O. Trainability of the prepubescent child. Phys. Sportsmed. 17(5):65-82, 1989.

3. Baumgartner, T.A., and S.S. Wood. Development of shoulder girdle strengthendurance in elementary children. Res. Q. 55(2):169-171, 1984.

4. Blimkie, C., D. MacDougal, D. Sale, E. Thonar, U. Smith, and S. Gasner. Resistance training, muscle morphology and contractile properties in prepubertal boys. Med. Sci. Sports Exer. 20:(suppl) 51, 1988.

5. Buller, A.J., J.C. Eccles, and R.M. Eccles. Differentiation of fast and slow muscles in the cat hind limb. J. Physiol. 150:399-416, 1960.

6. Buller, A.J., J.C. Eccles, and R.M. Eccles. Interactions between motorneurons and muscles in respect of the characteristic speeds of their responses. J. Physiol. 150:417-439, 1960.

7. Buller, A.J., and D.M. Lewis. Further observations on mammalian cross-innervated skeletal muscle. J. Physiol. 178:343-358, 1965.

8. Cheek, D.B., C.J. Migeon, and E.D. Mellits. The concept of biologic age. In: Human Growth-Body Composition, Cell Growth, Energy and Intelligence, D.B. Cheek (Ed.). Philadelphia: Lea \& Febiger, 1968, p. 246.

9. Clark, D.H., P. Vaccaro, and N.M. Andersen. Physiological alterations in 7- to 9-yearold boys following a season of competitive wrestling. Res. Q. 55(4):318-322, 1984. 
10. Close, R. Dynamic properties of fast and slow skeletal muscles of the rat during development. J. Physiol. 173:74-95, 1964.

11. Close, R. Force:velocity properties of mouse muscles. Nature, 206:718-719, 1965.

12. Close, R. Dynamic properties of fast and slow skeletal muscles in mammals. In: Exploratory Concepts in Muscular Dystrophy and Related Disorders, A.T. Milhorat (Ed.). Amsterdam: Exerpta Medica Foundation, 1967, pp. 142-150.

13. Docherty, D., H.A. Wenger, and M.L. Collis. The effects of resistance training on aerobic and anaerobic power of young boys. Med. Sci. Sports Exer. 19:389-392, 1987.

14. Duda, M. Prepubescent strength training gains support. Phys. Sportsmed., 14(2):157-161, 1986.

15. Edgerton, V.R. Exercise and the growth and development of muscle tissue. In: Physical Activity-Human Growth and Development, G.L. Rarick (Ed.). New York: Academic Press, 1973, pp. 1-31.

16. Ekblom, B. Effect of physical training in adolescent boys. J. Appl. Physiol. 27:350-355, 1969.

17. Fleck, S.J., and W.J. Kraemer. Designing Resistance Training Programs. Champaign, IL: Human Kinetics, 1987.

18. Ford, H.T., and J.R. Puckett. Competitive effects of prescribed weight-training and basketball programs on basketball skill test scores of ninth grade boys. Percept. Motor Skills, 56:23-26, 1983.

19. Garhammer, J. Strength Training: Your Ultimate Weight Conditioning Program. New York: Sports Illustrated Winner's Circle Books, Time Inc., 1987.

20. Guth, L. Trophic influences of nerve on muscle. Physiol. Rev. 48:645, 1968.

21. Hejna, W.F., A. Rosenberg, D.J. Buturusis, and A. Krieger. The prevention of sports injuries in high school students through strength training. Natl. Strength Cond. Assoc. J. 4(1):28-31, 1982.

22. Hetherington, M.R. Effect of isometric training on the elbow flexion force torque of grade five boys. Res. Q. 47(1):41-47, 1976.

23. Hooisma, J., D.W. Slaf, E. Meeter, and W.F. Stevens. Trophic support by neural explants of cultured muscle fibers. Exp. Neurol. 62:628, 1978.

24. Jacobson, S. Sequence of myelinization in the brain of the albino rat. A. Cerebral cortex, thalamus and related structures. J. Comp. Neurol. 121:5-29, 1963.

25. Kow, I.M., P.N. Wilkinson, and F.W. Chorrock. Axonal delivery of neuroplasmic components to muscle cells. Science, 155:342-345, 1967.

26. Kraemer, W.J. Endocrine responses to resistance exercise. Med. Sci. Sports Exer. 20(suppl): S152-S157, 1988.

27. Kraemer, W.J., and T.R. Baechle. Development of a strength training program. In: Sports Medicine (2nd edition), F.L. Allman and A.J. Ryan (Eds.). Orlando, Fl: Academic Press, 1989, pp. 113-127.

28. Kraemer, W.J., and S.J. Fleck. Resistance training: Exercise prescription. Phys. Sportsmed. 16(6):69-81, 1988.

29. Kraemer, W.J., S.J. Fleck, and M. Deschenes. A review: Factors in the exercise prescription of resistance training. Natl. Strength Cond. Assoc. 10:36-41, 1988.

30. Kraemer, W.J., B.J. Noble, B. Culver, and M.J. Clark. Physiologic responses to heavy-resistance exercise with very short rest periods. Int. J. Sports Med. 8:247-252, 1987.

31. Lentz, T.L. Nerve trophic function: In vitro assay of effects of nerve tissue on muscle cholinesterase activity. Science, 171:187-189, 1971. 
32. Lowrey, G.H. Growth and Development of Children. Chicago: Yearbook Medical Pub., 1973.

33. McGovern, N.B. Effects of circuit weight training on physical fitness of prepubescent children. Dissertation Abst. Int., 45:452-A, 1984.

34. Micheli, L.J. Pediatric and adolescent sports injury: Recent trends. Exer. Sport Sci. Rev. 14:359-374, 1986.

35. Micheli, L.J. Strength training in the young athlete. In: Competitive Sports for Children and Youth: An overview of research and issues, E.W. Brown and C.F. Crystal (Eds.). Champaign, IL: Human Kinetics, 1988, pp. 99-106.

36. National Strength and Conditioning Association. Position statement on prepubescent strength training. Nat. Strength Cond. Assoc. 7:27-31, 1985.

37. Nielsen, B., K. Nielsen, M. Behrendt-Hansen, and E. Asmussen. Training of "functional muscular strength" in girls 7-19 years old. In: Children and Exercise IX, K. Berg and B.O. Eriksson (Eds.). Baltimore: University Park Press, 1980, pp. 69-78.

38. Pearson, D.R., and D.L. Costill. The effects of constant external resistance exercise and isokinetic exercise training on work-induced hypertrophy. J. Appl. Sport Sci. Res. 2(3):39-41, 1988.

39. Pfeiffer R., and R.S. Francis. Effects of strength training on muscle development in prepubescent, pubescent and postpubescent males. Phys. Sportsmed. 14(9):134-143, 1986.

40. Rains, C.B., A. Weltman, B.R. Cahill, C.A. Janney, S.R. Tippett, and F.I. Katch. Strength training for prepubescent males: Is it safe? Am. J. Sports Med. 15(5):483-489, 1987.

41. Rauh, J.L., and D.A. Schumsky. Lean and non-lean body mass estimates in urban school children. In: Human Growth-Body Composition, Cell Growth, Energy and Intelligence, D.B. Cheek (Ed.). Philadelphia: Lea \& Febiger, 1968, p. 246.

42. Robbins, N., G. Karpati, and W.K. Engel. Histochemical and contractile properties in the cross-innervated guinea pig soleus muscle. Arch. Neurol. 20:318-329, 1969.

43. Rose, K.J. The Body in Time. New York: Wiley \& Sons, 1988, pp. 154-155.

44. Sailors, M., and K. Berg. Comparison of responses to weight training in pubescent boys and men. J. Sports Med. Phys. Fitness, 27:30-37, 1987.

45. Sale, D.G. Strength training in children. In: Perspectives in Exercise Science and Sports Medicine, C.V. Gisolfi and D.R. Lamb (Eds.). Carmel, IN: Benchmark Press, 1989, pp. 165-216.

46. Servedio, F.J., R.L. Bartels, and R.L. Hamlin. The effects of weight training using Olympic style lifts, on various physiological variables in prepubescent boys [abstract]. Med. Sci. Sports Exer. 17:288, 1985.

47. Sewall, L., and L.J. Micheli. Strength training for children. J. Pediatr. Orthoped. 6:143-146, 1986.

48. Shephard, R.J. Physical Activity and Growth. Chicago: Yearbook Medical Pub., 1982, p. 174.

49. Siegel, J. Fitness in prepubescent children: Implications for exercise training. Natl. Strength Cond. Assoc. J. 10(3):43-48, 1988.

50. Stanitski, C.L. Common injuries in preadolescent and adolescent athletes: Recommendations for prevention. Sports Med. 7:32-41, 1989.

51. Stone, M.H., and H.S. Obryant. Weight Training: A Scientific Approach. Minneapolis: Bellwether Press, 1987. 
52. Timiras, P.S. Developmental Physiology and Aging. New York: Macmillan, 1972, pp. 133-147.

53. Valladian, I., and D. Porter. Physical Growth and Development: From Conception to Maturity. Boston: Little, Brown \& Co., 1977, pp. 134-135.

54. Vrijens, J. Muscle strength development in the pre- and post-pubescent age. Med. Sports (Basel), 11:152-158, 1978.

55. Weltman, A. Weight training in prepubertal children. Physiologic benefits and potential damage. In: Advances in Pediatric Sport Sciences (Vol 3), O. Bar-Or (Ed.). Champaign, IL:Human Kinetics, 1989, pp. 101-130.

56. Weltman, A., C. Janney, C.B. Rians, K. Strand, B. Berg, S. Tippitt, J. Wise, B.R. Cahill, and F.I. Katch. The effects of hydraulic resistance strength training in prepubertal males. Med. Sci. Sports Exer. 18:629-638, 1986.

57. Weltman, A., C. Janney, C.B. Rians, K. Strand, and F.I. Katch. The effects of hydraulic-resistance strength training on serum lipid levels in prepubertal boys. Am. J. Dis. Child. 141(7):777-780, 1987.

58. Yakolev, P.I., and A.R. Lecours. The myelogenetic cycles of regional maturation of the brain. In: Regional Development of the Brain in Early Life, A. Minkowski (Ed.). Oxford: Blackwell Scientific Publ., 1967. 
Copyright of Pediatric Exercise Science is the property of Human Kinetics Publishers. Inc. and its content may not be copied or emailed to multiple sites or posted to a listserv without the copyright holder's express written permission. However, users may print, download, or email articles for individual use. 